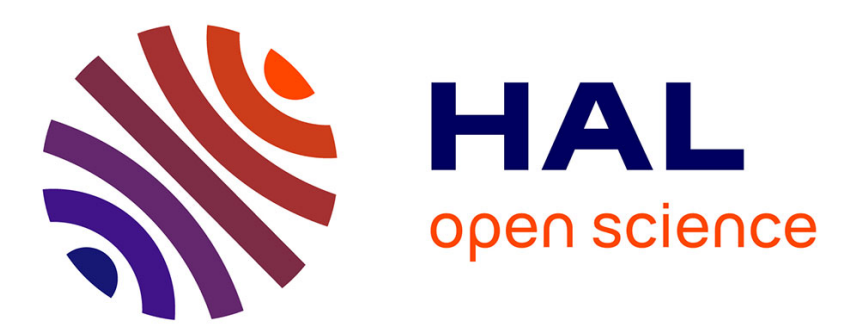

\title{
Weak field galvanomagnetic measurements to distinguish cubic from non-cubic environments
}

\author{
D.S. Kyriakos, N.A. Economou, R.S. Allgaier
}

\section{To cite this version:}

D.S. Kyriakos, N.A. Economou, R.S. Allgaier. Weak field galvanomagnetic measurements to distinguish cubic from non-cubic environments. Revue de Physique Appliquée, 1980, 15 (3), pp.733-739. 10.1051/rphysap:01980001503073300 . jpa-00244783

\section{HAL Id: jpa-00244783 \\ https://hal.science/jpa-00244783}

Submitted on 1 Jan 1980

HAL is a multi-disciplinary open access archive for the deposit and dissemination of scientific research documents, whether they are published or not. The documents may come from teaching and research institutions in France or abroad, or from public or private research centers.
L'archive ouverte pluridisciplinaire HAL, est destinée au dépôt et à la diffusion de documents scientifiques de niveau recherche, publiés ou non, émanant des établissements d'enseignement et de recherche français ou étrangers, des laboratoires publics ou privés. 


\title{
Weak field galvanomagnetic measurements to distinguish cubic from non-cubic environments
}

\author{
D. S. Kyriakos, N. A. Economou \\ Department of Physics, University of Thessaloniki, Greece
}

and R. S. Allgaier

Naval Surface Weapons Center, White Oak, Silver Spring, Maryland 20910, U.S.A.

(Reçu le 16 octobre 1979, révisé le 23 novembre 1979, accepté le 26 novembre 1979)

\begin{abstract}
Résumé. - Des procédures qui utilisent une extension de la technique Van der Pauw sont développées pour distinguer, par des mesures galvanomagnétiques de champ faible, des symétries cubiques et non cubiques. Nous considérons les couches orientées (001), (110) et (111) pour lesquelles les expressions analytiques sont données. Des échantillons plats, en forme de cercle peuvent être utilisés. Nous avons aussi trouvé que, en général, quand $\bar{B}=(B u, B v, B w)$ un comportement de torsion est attendu, tel que, les directions principales de la surface de résistivité et les axes crystallographiques se trouvent dans des directions différentes.
\end{abstract}

\begin{abstract}
Procedures utilizing an extension of the Van der Pauw technique are developed to distinguish by weak field galvanomagnetic measurements cubic and non-cubic symmetries. We consider (001), (110) and (111) oriented layers for which analytic expressions are given. Planar circular samples may be used. We have also found that in general when $\bar{B}=(B u, B v, B w)$ a skewed behaviour is expected such that the principal directions of the surface of the resistivity magnitude and the crystallographic axes lie in different directions.
\end{abstract}

1. Introduction. - Under weak field conditions the relation between the electric field $\bar{E}$ and the current density $\bar{J}$ in the presence of a magnetic field $\bar{B}$ may be expressed as [1]

$$
E_{i}=\rho_{i j} J_{j}+\rho_{i j k} J_{j} B_{k}+\rho_{i j k l} J_{j} B_{k} B_{l}+\cdots,
$$

where the galvanomagnetic (GVM) coefficients $\rho_{i j}$, $\rho_{i j k}$ and $\rho_{i j k l}$ are tensor elements of the zero field resistivity, the weak field Hall coefficient (WFHC) and the weak field magnetoresistance (WFMR). In (1) the implied summation is carried over all possible values of all repeated indices. Recently Allgaier et al. [2-5] have developed an extension of the SeitzPearson-Suhl [6] magnetoresistance formula applicable to (001) and (111) oriented layers in a situation where it has not been determined that the environment is, in fact, cubically symmetric. They showed how four WFMR measurements could be made on a single sample in either orientation, making it possible to distinguish between cubic and tetragonal environments in the first case, and among cubic, trigonal, and hexagonal ones in the second orientation.

In the present paper, we develop procedures for utilizing an extension [7] of the Van der Pauw technique [8] to distinguish cubic and non-cubic symmetries from weak-field galvanomagnetic measürements. We consider (001), (110) and (111) oriented layers.

In the case of cubic crystals [groups $\mathrm{O}_{h}(\mathrm{~m} 3 \mathrm{~m})$, $\mathrm{O}(432)$ and $T_{d}(43 \mathrm{~m})$ ], five different weak-field GVM coefficients need to be determined, namely

$$
\rho_{0}, \rho_{123}, \rho_{1111}, \rho_{1122} \text { and } \rho_{1212} \text {. }
$$

Thus the zero field resistivity $\rho_{0}$ and the WFHC $\rho_{123}$ are isotropic, while the relative values of the three WFMR coefficients are related to the types of bandstructure and scattering anisotropies which can occur within the framework of cubic symmetry.

2. The measuring principles. - The measurements are based on Wasscher's extension [7] of the method of Van der Pauw [8] to the case of crystals with an anisotropic resistivity. He describes how this extension might be applied to magnetoresistance measurements, i.e., to a magnetic-field induced resistivity anisotropy. We use a planar, circular sample with four contacts $\mathrm{ABCD}$ taken along the circumference on two perpen- 
dicular diameters (Fig. 1). Wasscher discusses the advantages of this configuration. We may then define the resistances

$$
R_{1}=\frac{V_{\mathrm{D}}-V_{\mathrm{C}}}{I_{\mathrm{AB}}}, \quad R_{2}=\frac{V_{\mathrm{A}}-V_{\mathrm{D}}}{I_{\mathrm{BC}}},
$$

and

$$
R_{12}=\frac{V_{\mathrm{A}}-V_{\mathrm{C}}}{I_{\mathrm{BD}}}
$$

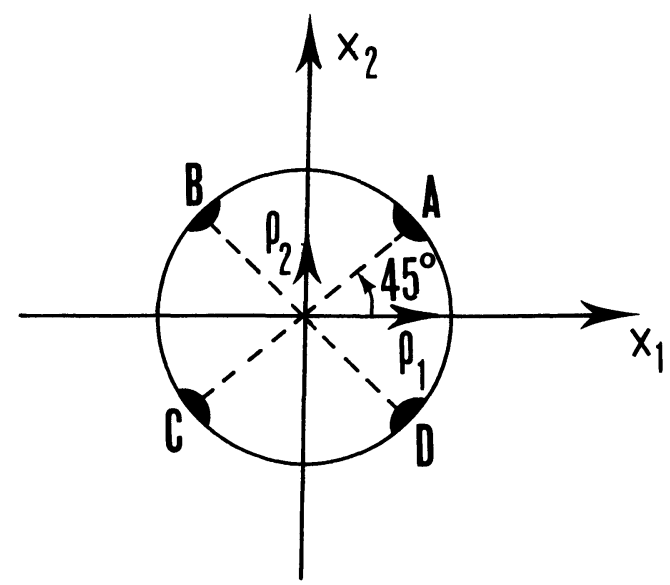

Fig. 1. - Planar anisotropic circular sample with contacts in the right position for the determination of $\rho_{1}$ and $\rho_{2}$.

The maximum value of the resistance ratio,

$$
\left(R_{1} / R_{2}\right)_{\max }=\left(R_{1}\right)_{\max } /\left(R_{2}\right)_{\min },
$$

is obtained when the contacts are placed at an angle of $45^{\circ}$ to the directions of the principal axes of resistivity $x_{1}$ and $x_{2}$ with resistivities $\rho_{11}=\rho_{1}$ and $\rho_{22}=\rho_{2}$ correspondingly $\left(\rho_{1}>\rho_{2}\right)$. This ratio is related to the anisotropy ratio $\lambda=\rho_{1} / \rho_{2}$. For the principal axis resistivities in the plane of the sample, we have [9]

$$
\rho_{1}=\frac{\lambda^{1 / 2} \pi d\left(R_{1}\right)_{\max }}{\ln \frac{2}{1-k}}
$$

and

$$
\rho_{2}=\frac{\lambda^{-1 / 2} \pi d\left(R_{2}\right)_{\min }}{\ln \frac{2}{1+k}}
$$

where $d$ is the thickness and $k$ is the modulus of the elliptic integral that is related to the ratio $\lambda[7,9]$. It should be mentioned here, that the above technique gives the same results as two independent measurements in the case of the classical procedure with $\bar{J}$ lying first in the $x_{1}$ direction and the other in the $x_{2}$ direction. In fact, from eq. (3) and figure 1 it is evident that the resistances $\left(R_{1}\right)_{\max }$ and $\left(R_{2}\right)_{\min }$ are defined with $\bar{J}$ lying in the directions $x_{1}$ and $x_{2}$ respectively.

The Hall coefficient in this method, for a field perpendicular to the plane of the sample, is determined from the relation [8]

$$
R_{\mathrm{H}}=\frac{d}{B} \Delta R_{12}
$$

where $\Delta R_{12}$ is the change in the resistance $R_{12}$ due to the presence of the magnetic field.

As it is well known [10], if the direction cosines of $\bar{J}$ and $\bar{B}$ are $p, q, r$ and $u, v, w$, respectively, then the zero-field resistivity in the direction of $\bar{J}$ is

$$
\rho(0)=\frac{\bar{E} . \bar{J}}{J^{2}}=\rho_{0} p^{2}+\rho_{0} q^{2}+\rho_{0} r^{2}=\rho_{0},
$$

while in the presence of the magnetic field $\bar{B}$ we have

$$
\begin{aligned}
\rho(\bar{B})= & \frac{\bar{E}(\bar{B}) \cdot \bar{J}}{J^{2}}= \\
= & {\left[\rho_{0}+\left(\rho_{1111} u^{2}+\rho_{1122} v^{2}+\rho_{1122} w^{2}\right) B^{2}\right] p^{2} } \\
& +\left[\rho_{0}+\left(\rho_{1122} u^{2}+\rho_{1111} v^{2}+\rho_{1122} w^{2}\right) B^{2}\right] q^{2} \\
& +\left[\rho_{0}+\left(\rho_{1122} u^{2}+\rho_{1122} v^{2}+\rho_{1111} w^{2}\right) B^{2}\right] r^{2} \\
& +4 \rho_{1212} B^{2}[u v p q+v w q r+w u r p] .
\end{aligned}
$$

Comparing the two eqs. (7) and (8), we conclude that the presence of magnetic field introduces an anisotropy in the specific resistivity such that its principal axes and the crystallographic axes, in general, lie in different directions [11]. The corresponding effect is known as magnetoresistance skewness [2, 12].

3. Sample parallel to the (001) plane. - In this case the sample plane contains the two axes $x_{1}$ and $x_{2}$, and we have $\bar{J}=(. J \cos \omega, J \sin \omega, 0)$ (Fig. 2). To obtain the GVM coefficients we perform measurements in the following manner.

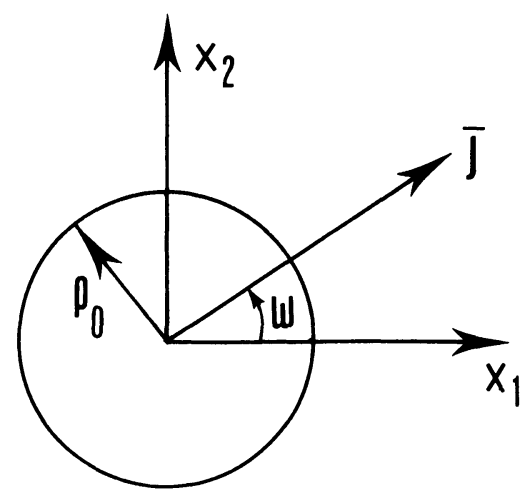

Fig. 2. - Polar distribution of $\rho$ on the $\left(x_{1}, x_{2}\right)$ plane.

3.1 SPECIFIC RESISTANCE. - From the equation

$$
\rho=\frac{\pi d}{\ln 2} \frac{R_{1}+R_{2}}{2} f\left(\frac{R_{1}}{R_{2}}\right)
$$

derived by Van der Pauw [8] we have

$$
\rho_{0}=\frac{\pi d}{\ln 2} \frac{R_{1}+R_{2}}{2}
$$


because for the contacts at right angles, $R_{1}=R_{2}$, and the corrective factor is $f(1)=1$.

3.2 Hall COefficient. - When $\bar{B}=(0,0, B)$, the components of the electric field are

$E_{1}=\rho_{123} J_{2} B, \quad E_{2}=-\rho_{123} J_{1} B$ and $E_{3}=0$.

Thus $\left|\bar{E}_{\mathrm{H}}\right|=\rho_{123} J B$ and $\rho_{123}$ is determined from eq. (6).

3.3 WFMR COEFFICIENTS. - Two configurations will be treated :

a) $\bar{B}=(B, 0,0)$. Using figure 2 and eq. (8) we obtain

$\rho(\bar{B})=\left(\rho_{0}+\rho_{1111} B^{2}\right) \cos ^{2} \omega+\left(\rho_{0}+\rho_{1122} B^{2}\right) \sin ^{2} \omega$,

corresponding to a resistivity anisotropy with principal axes lying in the crystallographic directions $x_{1}$ and $x_{2}$ (Fig. 3). By measuring the experimental quantities $\rho_{0}, \rho_{11}(\bar{B})$ and $\rho_{22}(\bar{B})$, we obtain

$$
\begin{aligned}
\Delta \rho & =\rho_{1111} B^{2} \cos ^{2} \omega+\rho_{1122} B^{2} \sin ^{2} \omega \\
& =\Delta \rho_{11} \cos ^{2} \omega+\Delta \rho_{22} \sin ^{2} \omega,
\end{aligned}
$$

where

$\Delta \rho_{11}=\rho_{11}(\bar{B})-\rho_{0}, \quad \Delta \rho_{22}=\rho_{22}(\bar{B})-\rho_{0}$.

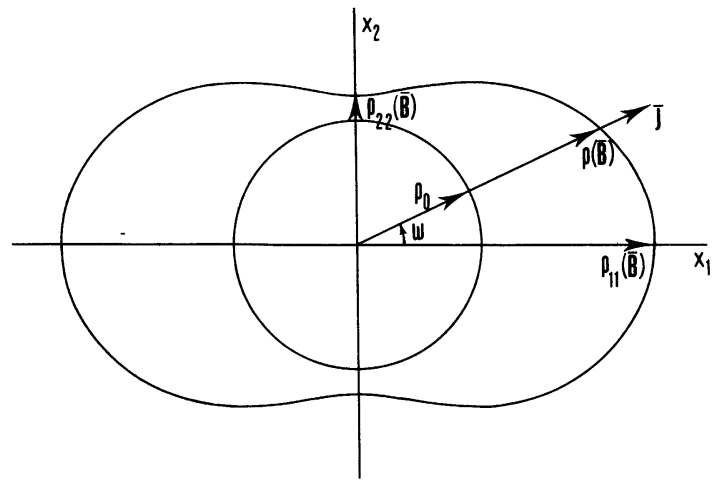

Fig. 3. - The anisotropy in resistance induced by the magnetic field.

Thus we are led to the relations

$$
\rho_{1111}=\frac{\Delta \rho_{11}}{B^{2}} \quad \text { and } \quad \rho_{1122}=\frac{\Delta \rho_{22}}{B^{2}}
$$

by which $\rho_{1111}$ and $\rho_{1122}$ may be determined from experimental measurements of $\Delta \rho_{11}$ and $\Delta \rho_{\underline{22}}$. It is evident that, with the above direction of $\bar{B}, \Delta \rho_{11}$ corresponds to the longitudinal magnetoresistance while $\Delta \rho_{22}$ corresponds to the transverse one [13], as stated previously in connection with the defining eqs. (4) and (5). It should be noted here that in the case of $\bar{B}=(0, B, 0)$, the same anisotropy appears with a phase difference of $90^{\circ}$ so that the same two WFMR coefficients, $\rho_{1111}$ and $\rho_{1122}$, are obtained, but in reverse order. Finally for $\bar{B}=(0,0, B)$ the sample remains isotropic and only the coefficient $\rho_{1122}$ can be determined.

This last case is interesting when we have (001)oriented films or surface layers. Suppose that the value of $\rho_{1122}$ as determined from this configuration is different from that determined by eq. (14). This result is an indication of tetragonal, rather than cubic symmetry, with the tetragonal axis lying along the [001] direction [4].

b) $\bar{B}=(B u, B v, 0)$. In this case an anisotropic (skewed) behaviour is expected, such that the principal axes and the crystallographic axes lie in different directions (Fig. 4): If these directions are at an angle $\varphi$ to each other then the following equation is valid (see appendix)

$$
4 \rho_{1212} B^{2} u v=\sin 2 \varphi\left[\rho_{11}^{\prime}(\bar{B})-\rho_{22}^{\prime}(\bar{B})\right]
$$

where $\rho_{11}^{\prime}(\bar{B}), \rho_{22}^{\prime}(\bar{B})$ are the principal resistivities under the influence of the magnetic field. In this way, the coefficient $\rho_{1212}$ is obtained. The procedure is to choose first a certain direction of $\bar{B}$ with respect to the $\left(x_{1}, x_{2}\right)$, set of axes and then rotate the system of contacts, without changing the direction of $\bar{B}$, until the maximum value of $R_{1} / R_{2}$ is found ; thus the value of the angle $\varphi$ and the resistances $\rho_{11}^{\prime}(\bar{B})$ and $\rho_{22}^{\prime}(\bar{B})$ are obtained.

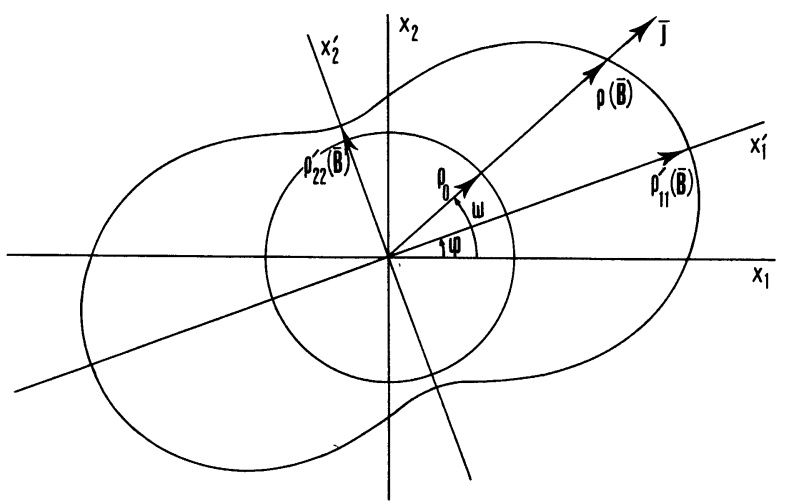

Fig. 4. - Determination of $\Delta \rho$ for anisotropy that has its principal axes inclined by an angle $\varphi$ to the crystallographic axes.

Eq. (15) leads to a new definition of the $\rho_{i j i j}$ coefficient. Thus for $u=v=1 / \sqrt{2}$ we have

$$
\rho_{i j i j}=\frac{1}{2 B^{2}} \sin 2 \varphi\left[\Delta \rho_{i i}^{\prime}(\bar{B})-\Delta \rho_{j j}^{\prime}(\bar{B})\right]
$$

where $\Delta \rho_{i i}^{\prime}(\bar{B})=\rho_{i i}^{\prime}(\bar{B})-\rho_{0}$ and $\Delta \rho_{j j}^{\prime}(\bar{B})=\rho_{j j}^{\prime}(\bar{B})-\rho_{0}$. Therefore the $\rho_{i j i j}$ coefficient expresses the difference between the two principal magnetoresistances and the imposed skewness by the magnetic field. 
4. Sample parallel to the (110) plane. - In the case that the specimen is parallel to a (110) plane as in the case of thin films grown on (110) substrates, a new set of axes should be chosen in terms of which the measurements may be defined. We use the coordinate system $[1 \overline{1} 0]=x_{1}^{\prime},[110]=x_{2}^{\prime}$ and $[001]=x_{3}^{\prime}$ (Fig. 5). With this set, the plane of the sample contains the $x_{1}^{\prime}$ and $x_{3}^{\prime}$ axes. Axes $x_{1}^{\prime}$ and $x_{2}^{\prime}$ exhibit two-fold, and $x_{3}^{\prime}$, fourfold rotational symmetry. These axes are similar to the axes of the tetragonal system for the groups $\mathrm{D}_{4}(422), \mathrm{C}_{4 \mathrm{v}}(4 \mathrm{~mm}), \mathrm{D}_{2 \mathrm{~d}}, \mathrm{~V}_{\mathrm{d}}(\overline{4} 2 \mathrm{~m})$, and $\mathrm{D}_{4 \mathrm{~h}}(4 / \mathrm{mmm})$.

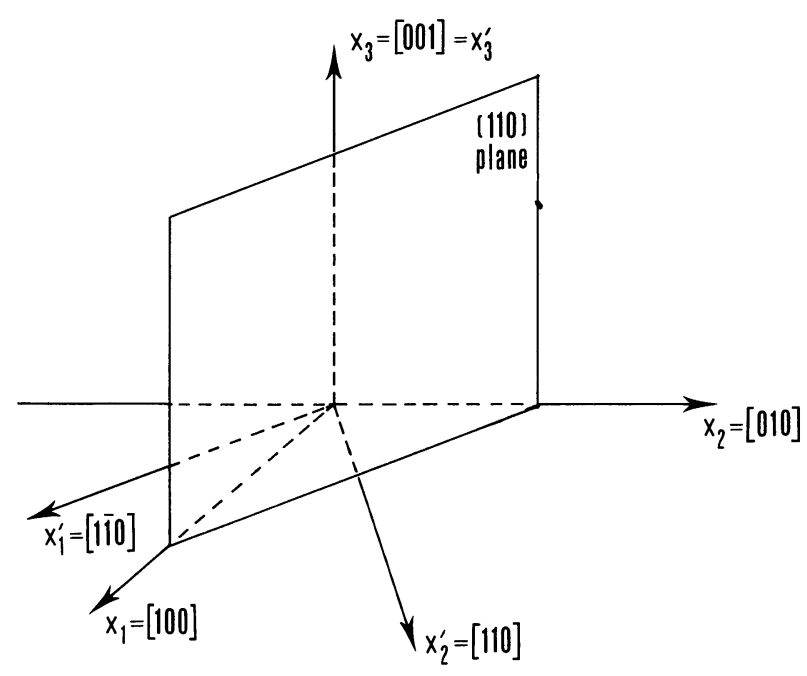

Fig. 5. - The coordinate system in the case of a sample parallel to the (110) plane.

The resistivity and Hall coefficients for these groups involve the four different nonzero tensor elements [13]

$$
\rho_{11}\left(=\rho_{22}\right), \rho_{33}, \rho_{123} \text { and } \rho_{231}\left(=\rho_{312}\right),
$$

while the nonzero WFMR coefficients are presented in table I, using the following correspondence :

$$
\begin{array}{lll}
11 \rightarrow 1 & 22 \rightarrow 2 & 33 \rightarrow 3 \\
23=32 \rightarrow 4 & 31=13 \rightarrow 5 & 12=21 \rightarrow 6 .
\end{array}
$$

In the case of cubic symmetry, we are seeking, by the transformation of the elements from the $x_{\mathrm{i}}$ to the

\begin{tabular}{|c|c|c|c|c|c|c|}
\hline & $B_{1}^{2}$ & $B_{2}^{2}$ & $B_{3}^{2}$ & $B_{2} B_{3}$ & $B_{3} B_{1}$ & $B_{1} B_{2}$ \\
\hline & - & - & - & - & - & \\
\hline$E_{1}, J_{1}$ & $\rho_{11}$ & $\rho_{12}$ & $\rho_{13}$ & 0 & 0 & 0 \\
\hline$E_{2}, J_{2}$ & $\rho_{12}$ & $\rho_{11}$ & $\rho_{13}$ & 0 & 0 & 0 \\
\hline$E_{3}, J_{3}$ & $\rho_{31}$ & $\rho_{31}$ & $\rho_{33}$ & 0 & 0 & 0 \\
\hline$E_{2}, J_{3}$ & 0 & 0 & 0 & $2 \rho_{44}$ & 0 & 0 \\
\hline$E_{3}, J_{1}$ & 0 & 0 & 0 & 0 & $2 \rho_{44}$ & 0 \\
\hline$E_{1}, J_{2}$ & 0 & 0 & 0 & 0 & 0 & $2 \rho_{66}$ \\
\hline
\end{tabular}

Table I. - The non-zero WFMR coefficient for the groups $\mathrm{D}_{4}, \mathrm{C}_{4 \mathrm{v}}, \mathrm{D}_{2 \mathrm{~d}}, \mathrm{~V}_{\mathrm{d}}, \mathrm{D}_{4 \mathrm{~h}}$. new set, the GVM coefficients presented in eq. (17) and in table I. Thus we have the following results

$$
\begin{aligned}
\rho_{11}^{\prime} & =\rho_{22}^{\prime}=\rho_{33}^{\prime}=\rho_{0} \\
\rho_{123}^{\prime} & =\rho_{231}^{\prime}=\rho_{312}^{\prime}=\rho_{123} \\
\rho_{1111}^{\prime} & =\rho_{2222}^{\prime}=\frac{1}{2}\left(\rho_{1111}+\rho_{1122}+2 \rho_{1212}\right) \\
\rho_{3333}^{\prime} & =\rho_{1111} \\
\rho_{1133}^{\prime} & =\rho_{2233}^{\prime}=\rho_{3311}^{\prime}=\rho_{3322}^{\prime}=\rho_{1122} \\
\rho_{2111}^{\prime} & =\rho_{1122}^{\prime}=\frac{1}{2}\left(\rho_{1111}+\rho_{1122}-2 \rho_{1212}\right) \\
\rho_{1212}^{\prime} & =\frac{1}{2}\left(\rho_{1111}+\rho_{1122}\right) \\
\rho_{2323}^{\prime} & =\rho_{1313}^{\prime}=\rho_{1212} .
\end{aligned}
$$

The difference between the cubic case, eqs. (18)-(25), and tetragonal symmetry, eq. (17) and table I, is obvious. The problem is thus reduced to the determination of the $\rho_{i j}(\bar{B})$ from measurements of the $\rho_{k l}^{\prime}(\bar{B})$, thereby to the deduction of the tetragonal environment in case of reduced symmetry. The procedure is as follows :

4.1 SPeCifiC ResistanCe and Hall COEFficient. - Using the method presented in 3.3.1-3.2, we measure $\rho_{11}^{\prime}=\rho_{33}^{\prime}=\rho_{0}$ and $\rho_{123}^{\prime}=\rho_{123}$. In case the actual symmetry is tetragonal, however, $\rho_{11}^{\prime} \neq \rho_{33}^{\prime}$. Thus by rotating the four contacts, a maximum in the ratio $R_{1} / R_{2}$ can be found, thus determining the anisotropy ratio $\lambda$. From eqs. (4) and (5), $\rho_{11}^{\prime}$ and $\rho_{33}^{\prime}$ are determined and the directions of the $x_{1}^{\prime}, x_{3}^{\prime}$ axes are defined. In this manner, the reduced symmetry is revealed and evaluated. It should also be mentioned that the measurement of the Hall coefficient with perpendicular magnetic field $[\bar{B}=(0, B, 0)]$ yields the coefficient $\rho_{231}^{\prime}=\rho_{312}^{\prime}$.

4.2 WFMR COEFFICIENTS. - In the case of $\bar{B}=(B u, B v, B w)$ and $\bar{J}=(. J p, 0, J r)$ (referred to the primed coordinate system), the anisotropy that appears has principal axes which, in general, are different from the $x_{1}^{\prime}$ and $x_{3}^{\prime}$ axes. For cubic symmetry, the measurement of $\rho_{1122}^{\prime}, \rho_{3311}^{\prime}\left(=\rho_{1133}^{\prime}\right)$, and $\rho_{3333}^{\prime}$ allows the determination of $\rho_{1111}, \rho_{1122}$, and $\rho_{1212}$ from the relations (21), (22) and (23). Thus

a) For $\bar{B}(0, B, 0)$ (that is, perpendicular to the sample plane) we have

$$
\rho(\bar{B})=\left[\rho_{11}^{\prime}+\rho_{1122}^{\prime} B^{2}\right] p^{2}+\left[\rho_{33}^{\prime}+\rho_{3311}^{\prime} B^{2}\right] r^{2}
$$

and

$\Delta \rho=\rho_{1122}^{\prime} B^{2} p^{2}+\rho_{3311}^{\prime} B^{2} r^{2}=\Delta \rho_{11}^{\prime} p^{2}+\Delta \rho_{33}^{\prime} r^{2}$,

where

$$
\Delta \rho_{11}^{\prime}=\rho_{11}^{\prime}(\bar{B})-\rho_{11}^{\prime}, \quad \Delta \rho_{33}^{\prime}=\rho_{33}^{\prime}(\bar{B})-\rho_{33}^{\prime} .
$$

This leads to

$$
\rho_{1122}^{\prime}=\frac{\Delta \rho_{11}^{\prime}}{B^{2}} \text { and } \rho_{3311}^{\prime}=\frac{\Delta \rho_{33}^{\prime}}{B^{2}} .
$$


b) For $\bar{B}(0,0, B)$ we have

$\rho(\bar{B})=\left[\rho_{11}^{\prime}+\rho_{1133}^{\prime} B^{2}\right] p^{2}+\left[\rho_{33}^{\prime}+\rho_{3333}^{\prime} B^{2}\right] r^{2}$,

which leads to

$$
\rho_{1133}^{\prime}=\frac{\Delta \rho_{11}^{\prime}}{B^{2}} \quad \text { and } \quad \rho_{3333}^{\prime}=\frac{\Delta \rho_{33}^{\prime}}{B^{2}} .
$$

The case of reduced, tetragonal symmetry is immediately apparent from these measurements if we find that

$$
\rho_{3311}^{\prime} \neq \rho_{1133}^{\prime} \text {. }
$$

5. Sample parallel to the (111) plane. - In many instances we deal with material that cleaves along the (111) plane. As in the previous section, we should again choose a sample-oriented coordinate system in terms of which the tensor elements are more conveniently defined and measured. As a new frame of reference we choose the axes $[1 \overline{1} 0]=x_{1}^{\prime}$ and $[11 \overline{2}]=x_{2}^{\prime}$ in the (111) plane and the [111] $=x_{3}^{\prime}$ perpendicular to the (111) plane (Fig. 6) as in [2] and [5]. The $x_{1}^{\prime}, x_{2}^{\prime}$ and $x_{3}^{\prime}$ directions coincide with the two-fold axis, the bisectrix and the three-fold axis, respectively, of trigonal crystals $\left[\mathrm{C}_{3 \mathrm{v}}(3 \mathrm{~m})\right.$ and $\mathrm{D}_{3 \mathrm{~d}}(\overline{3} \mathrm{~m})$ groups].

The nonzero elements of the resistivity and Hall tensors for these groups are $[1,13]$

$$
\rho_{11}\left(=\rho_{22}\right), \quad \rho_{33}, \quad \rho_{123} \text { and } \rho_{231}\left(=\rho_{312}\right),
$$

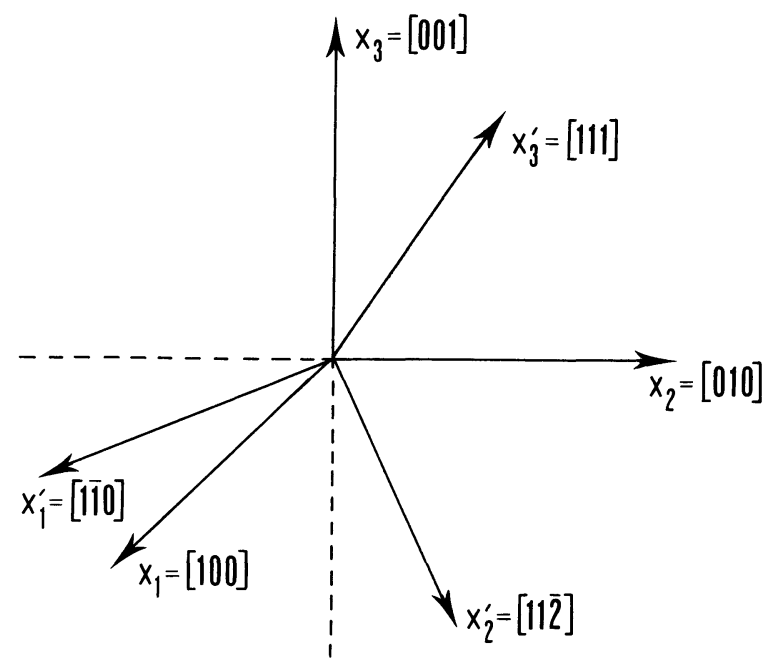

Fig. 6. - The coordinate system in the case of a sample parallel to the (111) plane.

while the nonzero WFMR coefficients are given in table II with the following corespondence :

$$
\begin{gathered}
11 \rightarrow 1 \quad 22 \rightarrow 2 \quad 33 \rightarrow 3 \quad 23=32 \rightarrow 4 \\
13=31 \rightarrow 5 \quad 12=21 \rightarrow 6 .
\end{gathered}
$$

\begin{tabular}{|c|c|c|c|c|c|c|}
\hline & $B_{1}^{2}$ & $B_{2}^{2}$ & $B_{3}^{2}$ & $B_{2} B_{3}$ & $B_{3} B_{1}$ & $B_{1} B_{2}$ \\
\hline & - & - & - & - & - & - \\
\hline$E_{1}, J_{1}$ & $\rho_{11}$ & $\rho_{12}$ & $\rho_{13}$ & $2 \rho_{14}$ & 0 & 0 \\
\hline$E_{2}, J_{2}$ & $\rho_{12}$ & $\rho_{11}$ & $\rho_{13}$ & $-2 \rho_{14}$ & 0 & 0 \\
\hline$E_{3}, J_{3}$ & $\rho_{31}$ & $\rho_{31}$ & $\rho_{33}$ & 0 & 0 & 0 \\
\hline$E_{2}, J_{3}$ & $\rho_{41}$ & $-\rho_{41}$ & 0 & $2 \rho_{44}$ & 0 & 0 \\
\hline$E_{3}, J_{1}$ & 0 & 0 & 0 & 0 & $2 \rho_{44}$ & $2 \rho_{41}$ \\
\hline$E_{1}, J_{2}$ & 0 & 0 & 0 & 0 & $2 \rho_{14}$ & $\rho_{11}-\rho_{1}$ \\
\hline
\end{tabular}

REVUE DE PHYSIQUe APPLIQUÉE. - T. 15, No 3 , MARS 1980
Table II. - The non-zero WFMR coefficient for the groups $\mathrm{C}_{3 \mathrm{v}}$ and $\mathrm{D}_{3 \mathrm{~d}}$.

Returning now to the cubic case. With the transformation from the $x_{i}$ to the $x_{i}^{\prime}$ coordinates we are seeking the GVM coefficients of eq. (33) and table II. Thus we have the following results

$$
\begin{aligned}
\rho_{11}^{\prime} & =\rho_{22}^{\prime}=\rho_{33}^{\prime}=\rho_{0} \\
\rho_{123}^{\prime} & =\rho_{231}^{\prime}=\rho_{312}^{\prime}=\rho_{123} \\
\rho_{1111}^{\prime} & =\rho_{2222}^{\prime}=\frac{1}{2}\left(\rho_{1111}+\rho_{1122}+2 \rho_{1212}\right) \\
\rho_{3333}^{\prime} & =\frac{1}{3}\left(\rho_{1111}+2 \rho_{1122}+4 \rho_{1212}\right) \\
\rho_{1122}^{\prime} & =\rho_{2211}^{\prime}=\frac{1}{6}\left(\rho_{1111}+5 \rho_{1122}-2 \rho_{1212}\right) \\
\rho_{1133}^{\prime} & =\rho_{3311}^{\prime}=\rho_{2233}^{\prime}=\rho_{3322}^{\prime} \\
& =\frac{1}{3}\left(\rho_{1111}+2 \rho_{1122}-2 \rho_{1212}\right) \\
\rho_{2323}^{\prime} & =\rho_{3131}^{\prime}=\frac{1}{3}\left(\rho_{1111}-\rho_{1122}+\rho_{1212}\right) \\
\rho_{1123}^{\prime} & =-\rho_{2223}^{\prime}=\rho_{1231}^{\prime}=\rho_{2311}^{\prime}=-\rho_{2322}^{\prime} \\
& =\rho_{3112}^{\prime}=\frac{\sqrt{2}}{6}\left(\rho_{1111}-\rho_{1122}-2 \rho_{1212}\right)
\end{aligned}
$$

and

$\rho_{1212}^{\prime}=\frac{1}{2}\left(\rho_{1111}^{\prime}-\rho_{1122}^{\prime}\right)$.

The difference between cubic and trigonal symmetry is obvious from a comparison of eqs. (34)-(42) with eq. (33) and table II. The problem is again reduced to the determination of the $\rho_{i j}(\bar{B})$ by measuring the $\rho_{k l}^{\prime}(\bar{B})$ and, in the case of reduced symmetry, of determining the trigonal environment. The procedure to be followed is outlined below.

5.1 SpeCific Resistance and Hall COEFFicient. - By using the method presented in 3.1-3.2, we measure the $\rho_{11}^{\prime}=\rho_{22}^{\prime}=\rho_{0}$ and $\rho_{123}^{\prime}=\rho_{123}$. If there is an environment of trigonal symmetry it cannot. be detected here, since on a specimen with faces parallel to the $\left(x_{1}^{\prime}, x_{2}^{\prime}\right)[=(111)]$ plane, the measurements of $\rho_{33}^{\prime}$ and $\rho_{231}^{\prime}$, are impossible.

5.2 WFMR COEFFICIENTS. - If $\bar{B}=(B u, B v, B w)$ and $\bar{J}=(J p, J q, 0)$ (in the primed system of reference), the resistivity anisotropy will appear with the principal axes different from the $x_{1}^{\prime}$ and $x_{2}^{\prime}$ directions. For cubic symmetry the measurement of $\rho_{1111}^{\prime}, \rho_{1122}^{\prime}$, and $\rho_{1133}^{\prime}$ allows the determination of $\rho_{1111}, \rho_{1122}$, and $\rho_{1212}$ from the relations (36), (38) and (39). Now we consider the specific cases 
a) $\bar{B}=(B, 0,0)$. Then

$\rho(\bar{B})=\left(\rho_{11}^{\prime}+\rho_{1111}^{\prime} B^{2}\right) p^{2}+\left(\rho_{22}^{\prime}+\rho_{1122}^{\prime} B^{2}\right) q^{2} ;$

that is, the anisotropy axes are $x_{1}^{\prime}$ and $x_{2}^{\prime}$, so that

$\Delta \rho=\rho_{1111}^{\prime} B^{2} p^{2}+\rho_{1122}^{\prime} B^{2} q^{2}=\Delta \rho_{11}^{\prime} p^{2}+\Delta \rho_{22}^{\prime} q^{2}$,

where

$$
\Delta \rho_{11}^{\prime}=\rho_{11}^{\prime}(\bar{B})-\rho_{11}^{\prime} \quad \text { and } \quad \Delta \rho_{22}^{\prime}=\rho_{22}^{\prime}(\bar{B})-\rho_{22}^{\prime} \text {. }
$$

Therefore

$$
\rho_{1111}^{\prime}=\frac{\Delta \rho_{11}^{\prime}}{B^{2}} \quad \text { and } \quad \rho_{1122}^{\prime}=\frac{\Delta \rho_{22}^{\prime}}{B^{2}} .
$$

b) $\bar{B}=(0,0, B)$. Then

$$
\begin{aligned}
\rho(\bar{B}) & =\left(\rho_{11}^{\prime}+\rho_{1133}^{\prime} B^{2}\right) p^{2}+ \\
& +\left(\rho_{22}^{\prime}+\rho_{1133}^{\prime} B^{2}\right) q^{2}=\rho_{11}^{\prime}+\rho_{1133}^{\prime} B^{2},
\end{aligned}
$$

which means that this case is isotropic $\left(\rho_{11}^{\prime}=\rho_{22}^{\prime}\right)$. Therefore

$$
\rho_{1133}^{\prime}=\frac{\rho(\bar{B})-\rho_{11}^{\prime}}{B^{2}}
$$

c) If the symmetry is cubic, then six different magnitudes are involved in the WFMR coefficients, eqs. (36) to (40) (only three are independent), while in the case of trigonal symmetry eight become independent, with the new inequalities $\rho_{3311}^{\prime} \neq \rho_{1133}^{\prime}$ and $\rho_{2311}^{\prime} \neq \rho_{1123}^{\prime}$. Therefore by calculating a fourth coefficient, e.g. the $\rho_{1123}^{\prime}$ from the relation (41), using $\rho_{1111}, \rho_{1122}$ and $\rho_{1212}$, and then measuring experimentally the $\rho_{1123}^{\prime}$, we have a way of distinguishing the two environments $[2,5]$.

To measure the $\rho_{123}^{\prime}$, we use $\bar{B}=(B u, B v, B w)$. As in $3.3 b$ ) the anisotropy axes form an angle $\varphi$ with $x_{1}^{\prime}$ and $x_{2}^{\prime}$. Thus

$$
\begin{aligned}
& \Delta \rho=\left[\rho_{11}^{\prime \prime}(\bar{B}) \cos ^{2} \varphi+\rho_{22}^{\prime \prime}(\bar{B}) \sin ^{2} \varphi-\rho_{11}^{\prime}\right] p^{2}+ \\
& \quad+\left[\rho_{11}^{\prime \prime}(\bar{B}) \sin ^{2} \varphi+\rho_{22}^{\prime \prime}(\bar{B}) \cos ^{2} \varphi-\rho_{22}^{\prime}\right] q^{2} \\
& \quad+\sin 2 \varphi\left[\rho_{11}^{\prime \prime}(\bar{B})-\rho_{22}^{\prime \prime}(\bar{B})\right] p q .
\end{aligned}
$$

In this case the following equations are valid

$$
\begin{array}{r}
\left(\rho_{1111}^{\prime} u^{2}+\rho_{1122}^{\prime} v^{2}+\rho_{1133}^{\prime} w^{2}+2 \rho_{1123}^{\prime} v w\right) B^{2}= \\
=\rho_{11}^{\prime \prime}(\bar{B}) \cos ^{2} \varphi+\rho_{22}^{\prime \prime}(\bar{B}) \sin ^{2} \varphi-\rho_{11}^{\prime}, \quad(50) \\
\left(\rho_{1122}^{\prime} u^{2}+\rho_{1111}^{\prime} v^{2}+\rho_{1133}^{\prime} w^{2}-2 \rho_{1123}^{\prime} v w\right) B^{2}= \\
=\rho_{11}^{\prime \prime}(\bar{B}) \sin ^{2} \varphi+\rho_{22}^{\prime \prime}(\bar{B}) \cos ^{2} \varphi-\rho_{22}^{\prime}, \quad(51)
\end{array}
$$

and

$$
\begin{aligned}
& 2\left[2 \rho_{1123}^{\prime} w u+\left(\rho_{1111}^{\prime}-\rho_{1122}^{\prime}\right) u v\right] B^{2}= \\
& =\sin 2 \varphi\left[\rho_{11}^{\prime \prime}(\bar{B})-\rho_{22}^{\prime \prime}(\bar{B})\right],
\end{aligned}
$$

where now the $\rho_{i i}^{\prime \prime}(\bar{B})$ are along the new axes of anisotropy.

Since we have already determined $\rho_{1111}^{\prime}, \rho_{1122}^{\prime}$ and $\rho_{1133}^{\prime}$, eqs. (50), (51) and (52) identify three possible methods to measure $\rho_{123}^{\prime}$. The experimental procedure is similar to that presented in $3.3 \mathrm{~b}$. To simplify matters, we may choose all the angles $45^{\circ}$ so that $u=1 / 2, v=1 / 2, w=1 / \sqrt{2}$.

6. Discussion and conclusions. - In this paper we have summarized the procedures for determining the WFGM coefficients in the case of cubically symmetric environments, by using the Van der Pauw experimental arrangement. Thus a reliable set of data may be obtained which allows to distinguish different environments in the case of (001), (110) and (111) oriented surface layers, revealing possible position dependent or structural anisotropies, and also allowing to follow phase transformations.

The analysis presented here is more advantageous or complementary with the classical one, based on the Seitz-Pearson-Suhl (SPS) formulation. This last procedure may prove in some cases difficult or impossible to apply. This is because in our case the current density $\bar{J}$ does not need to be in any particular direction, while in the classical experimental configuration only specific directions can be used and this in the case of specific crystallographic environments e.g. the SPS formulation does not apply in cases of reduced symmetry. Thus with the classical configuration only four WFMR coefficients can be determined [5], while with the proposed procedure the number is increased e.g. in the case of orthorhombic symmetry from one sample seven different WFMR coefficients are determined [11]. The advantage is obvious because elements concerning the band structure of the material may be calculated, such as the effective masses, the constants of the relaxation times and also the angle $\theta$ which defines the arrangement of the energy ellipsoids in $k$ space in a selfconsistent way.

Finally with this method the results have more physical sence i.e. in the case of the polar plotts of $\rho(0)$ and $\rho(\bar{B})$ (eqs. (7) and (8)). Thus the influence of the magnetic field in inducing resistance anisotropy is immediately apparent.

In conclusion we may say that the experimental method presented here is more simple and the results obtained are more informative. Application of this method to investigate the transformation of $\mathrm{SnTe}$ and $\mathrm{GeSnTe}$ and to define the environment after the transformation, which is a debated subject, is under consideration.

Appendix. Derivation of equation (15). - Refering to figure 4 , the transformation matrix in the new axes $x_{1}^{\prime}, x_{2}^{\prime}$, and $x_{3}^{\prime}$, is

$$
\left[\alpha_{i j}\right]=\left(\begin{array}{ccc}
\cos \varphi & -\sin \varphi & 0 \\
\sin \varphi & \cos \varphi & 0 \\
0 & 0 & 1
\end{array}\right),
$$


and thus the tensor element $\left[\rho_{i j}(\bar{B})\right]$ in the unprimed coordinate system is obtained from the relation

$$
\left[\rho_{i j}(\bar{B})\right]=\left[\alpha_{i k}\right]\left[\rho_{k l}^{\prime}(\bar{B})\right]\left[\alpha_{l j}\right]^{t},
$$

where $\left[\alpha_{l j}\right]^{t}$ is the transpose matrix of $\left[\alpha_{l j}\right]$. From this relation we have

$$
\left[\rho_{i j}(\bar{B})\right]=\left(\begin{array}{ccc}
\cos ^{2} \varphi \rho_{11}^{\prime}(\bar{B})+\sin ^{2} \varphi \rho_{22}^{\prime}(\bar{B}) & \frac{1}{2} \sin 2 \varphi\left[\rho_{11}^{\prime}(\bar{B})-\rho_{22}^{\prime}(\bar{B})\right] & 0 \\
\frac{1}{2} \sin 2 \varphi\left[\rho_{11}^{\prime}(\bar{B})-\rho_{22}^{\prime}(\bar{B})\right] & \sin ^{2} \varphi \rho_{11}^{\prime}(\bar{B})+\cos ^{2} \varphi \rho_{22}^{\prime}(\bar{B}) & 0 \\
0 & 0 & \rho_{33}(\bar{B})
\end{array}\right)
$$

where $\rho_{11}^{\prime}(\bar{B})$ and $\rho_{22}^{\prime}(\bar{B})$ are the principal resistivities along the $x_{1}^{\prime}$ and $x_{2}^{\prime}$ axes respectively. The resistivity in the direction of $\bar{J}$ in the presence of the field is

$$
\begin{aligned}
\rho(\bar{B})= & \rho_{i j}^{\prime}(B) \cos \omega_{i} \cos \omega_{j} \\
= & {\left[\rho_{11}^{\prime}(\bar{B}) \cos ^{2} \varphi+\rho_{22}^{\prime}(\bar{B}) \sin ^{2} \varphi\right] \cos ^{2} \omega+\left[\rho_{11}^{\prime}(\bar{B}) \sin ^{2} \varphi+\right.} \\
& \left.\quad+\rho_{22}^{\prime}(\bar{B}) \cos ^{2} \varphi\right] \sin ^{2} \omega+2 \frac{1}{2} \sin 2 \varphi\left[\rho_{11}^{\prime}(\bar{B})-\rho_{22}^{\prime}(\bar{B})\right] \cos \omega \sin \omega .
\end{aligned}
$$

Comparing eqs. (8) and (A.4) with $p=\cos \omega$, $q=\sin \omega$ and $r=0$ the following equations are obtained

$$
\begin{aligned}
\rho_{0}+\left(\rho_{1111} u^{2}+\rho_{1122} v^{2}\right) B^{2}= \\
=\rho_{11}^{\prime}(\bar{B}) \cos ^{2} \varphi+\rho_{22}^{\prime}(\bar{B}) \sin ^{2} \varphi
\end{aligned}
$$

$$
\begin{aligned}
& \rho_{0}+\left(\rho_{1122} u^{2}+\rho_{1111} v^{2}\right) B^{2}= \\
& =\rho_{11}^{\prime}(\bar{B}) \sin ^{2} \varphi+\rho_{22}^{\prime}(\bar{B}) \cos ^{2} \varphi \\
& 4 \rho_{1212} B^{2} u v=\sin 2 \varphi\left[\rho_{11}^{\prime}(\bar{B})-\rho_{22}^{\prime}(\bar{B})\right] .
\end{aligned}
$$

In a similar manner eqs. (50), (51) and (52) can be obtained for the case of a sample parallel to (111) plane.

\section{References}

[1] BEER, A. C., Galvanomagnetic Effects in Semiconductors (Academic Press, London) 1963.

[2] Allgaier, R. S., Restorff, J. B. and Bland Houston, Appl. Phys. Lett. 34 (1979) 158.

[3] Allgaier, R. S., Bland Houston and Restorff, J. B., J. Appl. Phys. 49 (1978) 4442.

[4] Allgaier, R. S. and Restorff, J. B., J. Appl. Phys. 50 (1979) 402.

[5] Allgaier, R. S., Restorff, J. B. and Bland Houston, Phys. Rev. B. 19 (1979) 6155.

[6] Seitz, F., Phys. Rev. 79 (1950) 372. Pearson, G. L. and Suhl, H., Phys. Rev. 83 (1951) 768.
[7] Wasscher, J. D., Philips Res. Rep., Suppl. 8 (1969) 1.

[8] Van der Pauw, L. J., Philips Res. Rep. 13 (1958) 1.

[9] Kyriakos, D. S. and Economou, N. A., Annal Sci., Fac. Phys. and Mathem. Univ. Thessaloniki (to be published).

[10] Nye, J. F., Physical Properties of Crystals (Oxford Univ. Press, London) 1967.

[11] Kyriakos, D. S. and Economou, N. A., Phys. Status Solidi (b) 94 (1979) 549.

[12] Kyriakos, D. S. and Economou, N. A., Appl. Phys. Lett. (in press).

[13] Smith, A. C., Janak, J. F. and AdleR, R. B., Electronic Conduction in Solids, McGraw-Hill (New York) 1967. 